\title{
MicroRNA-605 functions as a tumor suppressor by targeting INPP4B in melanoma
}

\author{
LAN CHEN, YAXUAN CAO, DONGYUN RONG, YE WANG and YU CAO \\ Department of Dermatology, The Affiliated Hospital of Guiyang Medical University, \\ Yunyan, Guiyang, Guizhou 550004, P.R. China
}

Received November 28, 2016; Accepted May 10, 2017

DOI: $10.3892 /$ or.2017.5740

\begin{abstract}
MicroRNAs (miRNAs) play crucial roles in the initiation and progression of various cancers, including melanoma. Recently, the genetic variants and deregulation of miR-605 have been reported to participate in carcinogenesis. However, the expression status of the miR-605 in melanoma tissues and its role in melanoma progression remain unknown. In this study, we found that miR-605 was significantly downregulated in melanoma cell lines and clinical specimens. Further function studies demonstrated that miR-605 suppressed melanoma cell growth both in vitro and in vivo. Moreover, INPP4B gene was identified as a target of miR-605 through bioinformatics analysis and luciferase reporter assays. Further analysis demonstrated that the inhibition of INPP4B mediated SGK3 activation was required for the suppressive role of miR-605 on melanomas cell growth. Collectively, our data suggest that miR-605 functions as a tumor suppressor by negatively regulating INPP4B mediated SGK3 activation in melanoma and may present a potential target for therapeutic intervention.
\end{abstract}

\section{Introduction}

Melanoma, a cancer derived from melanocytes, is the most aggressive skin cancer and responsible for $80 \%$ of skin cancerrelated deaths (1). According to a report from American Cancer Society, there are an estimated 76,380 new cases of melanoma and 10,130 melanoma-related deaths in the United States in 2016 (2). In China, it is estimated that approximately 3,200 Chinese individuals died from melanoma in 2015 (3). The

Correspondence to: Professor Yu Cao, Department of Dermatology, The Affiliated Hospital of Guiyang Medical University, 28 Guiyi Street, Yunyan, Guiyang, Guizhou 550004, P.R. China E-mail: caoyudoctor1@aliyun.com

Abbreviations: miRNAs, microRNAs; miR-605, microRNA-605; 3'-UTR, the 3'-untranslated region

Key words: miR-605, melanoma, INPP4B, SGK3 identification of activating mutations in BRAF has led to the development of targeted therapies to treat melanoma patients bearing these mutations. However, although patients exhibit an initial response to targeted therapies, a majority of them finally develop recurrences due to onset of acquired drug resistance (4). Therefore, there is an urgent need to elucidate the molecular basis of melanoma development and progression to facilitate the development of an effective strategy to treat this disease.

INPP4B was initially characterized as an inositol polyphosphate phosphatase hydrolyzing PtdIns(3,4)P2 to PtdIns(3)P. As the intracellular $\mathrm{PI}(3,4) \mathrm{P} 2$ is required for the full activation of Akt, a critical oncogene in various cancers, INPP4B can restrain the PI3K/Akt signaling (5). Recently, INPP4B was shown to play a tumor suppressor role in variety of cancers, including lung, prostate, bladder and melanocytic cancers (6-9). The tumor suppressive mechanism of INPP4B has been attributed to its negative regulation role in PI3K/Akt signaling. Nevertheless, increasing evidence is accumulating that INPP4B has an oncogene role. INPP4B was reported to be overexpressed in acute myeloid leukemia (AML) and high levels of INPP4B are predictive of poor clinical outcome and chemoresistance (10). In a subset of breast cancers with low Akt, INPP4B mediated SGK3 activation drives tumorigenesis $(11,12)$. These studies demonstrate that the role in carcinogenesis is cell type- and context-dependent. In melanoma, one recent study showed that INPP4B is overexpressed in melanoma tissue and functions as an oncogenic driver through activating SGK3 kinase (13). SGK3, a member of the AGC family of kinases, possesses certain shared substrates with Akt and is also activated by PI3K involving PDK1 and mTORC2 (14). Similar to Akt, abnormal activation of SGK3 is implicated in the induction and progression of multiple cancers, such as breast cancer, prostate cancer, glioblastoma and hepatocellular carcinoma (15-18). More recently, SGK3 was reported to contribute to the development of melanoma as a key mediator of PDK1 activity (19).

MicroRNAs (miRNAs), a class of small non-coding RNAs, can modulate gene expression at the post-transcription level by enhancing mRNA degradation or repressing mRNA translation through binding to the 3'-untranslated region (3'-UTR) of its target mRNA. Accumulating evidence demonstrates that miRNAs participate in various cellular processes, including cell proliferation, migration, apoptosis and differentiation (20). Due to the crucial role of miRNAs in these 
biological processes, their aberrant expression is involved in the initiation and progression of numerous cancers. miRNAs can function as either oncogenes or tumor suppressors, depending on their target genes. In human melanoma, oncogenic miRNAs, such as miR-148, miR-182 and miR-221, were reported to be upregulated in melanoma and increase tumor progression (21-23). In contrast, miRNAs, including miR-101, miR-137 and miR-200c, are downregulated in melanoma and exhibit tumor-suppressive roles (24-26).

miR-605 was first identified as a positive regulator of p53 activity through repressing the expression of $\mathrm{mdm} 2$, which targets p53 for degradation (27). Growing evidence demonstrate that the genetic variants or deregulation of miR-605 participates in carcinogenesis. The genetic variants of miR-605 has been reported to be associated with the susceptibility of various cancers, including gastric cancer, prostate cancer and lung cancer (28-30). miR-605 was shown to be downregulated in intrahepatic cholangiocarcinoma specimens and repress tumor progression by directly targeting PSMD10 (31). However, the role of miR-605 in melanoma is still unknown. In this study, we provide evidence that miR-605 is downregulated in melanoma cells and tissues and suppresses melanoma cell growth and tumorigenesis. Moreover, we show that the tumor suppressor role of miR-605 is mediated by its direct repression of INPP4B expression, which leads to the inactivation of SGK3 kinase.

\section{Materials and methods}

Antibodies and reagents. Antibodies against NDRG1, pNDRG1 (T346) and pSGK3 (Thr320) were purchased from Cell Signaling Technology (Beverly, MA, USA). Antibodies from Santa Cruz Biotechnology (Santa Cruz, CA, USA) included INPP4B, SGK3 and tubulin. Chemically synthesized miR-605 mimics and antagomiR-605 were obtained from RiboBio (Guangzhou, China).

Tissue samples. This study was approved by the ethics committee of the Affiliated Hospital of Guiyang Medical University. Tissue samples used for this study were obtained with informed consent from the participants at the affiliated hospital of Guiyang Medical University. Pathology of the tissue samples was confirmed, and then frozen in liquid nitrogen.

Cells and cell culture. HEMn-MP cells were cultured in Medium 254 (Cascade Biologics, Portland, OR, USA) containing human melanocyte growth supplement (HMGS; Life Technologies, Carlsbad, CA, USA). All the melanoma cell lines were maintained in Dulbecco's modified Eagle's medium (DMEM; Invitrogen, Carlsbad, CA, USA) containing 5\% fetal calf serum (FCS; Gibco-Life Technologies). Cells were grown in a $37^{\circ} \mathrm{C}$ incubator at $5 \% \mathrm{CO}_{2}$.

To generate Mel-RM cells stably overexpressing miR-605, cells were infected with Lentivirus-harboring hsa-miR-605 (GeneChem Co., Shanghai, China) and selected by treatment with $1 \mu \mathrm{g} / \mathrm{ml}$ puromycin (Sigma-Aldrich, St. Louis, MO, USA) for 10 days.

Quantitative real-time PCR. miRNA was isolated using a mirVana miRNA isolation kit (Ambion, Carlsbad, CA, USA) according to the manufacturer's suggestions. Expression of miR-605 was measured with the PrimeScript miRNA RT-PCR kit (Takara, Dalian, China) and the level of U6 was used as an endogenous control. The forward primer for miR-605 is: 5'-TGCGGTAAATCCCATGGTGCCTTC-3'; the reverse primer for miRNAs is the UnimiRqPCR Primer (Takara). The forward primer for U6 is: 5'-GCGCGTCGTGAAGCGTTC-3'; the reverse primer for U6 is 5'-GTGCAGGGTCCGAGGT-3'.

Total RNA was extracted using TRIzol (Invitrogen, Grand Island, NY, USA) and reverse-transcribed into cDNA with M-MLV Reverse Transcriptase (Promega, Madison, WI, USA). The qRT-PCR was performed in an ABI 7500 system using a Takara SYBR RT-PCR kit (Takara) and the level of $\beta$-actin mRNA was used as an endogenous control. The primer sequences were as follows: INPP4B (forward: 5'-CCCCGG GTACTGAGGCTTCG-3'; reverse: 5'-CTTTGTATTCTCTC CCGGAGGCG-3'); $\beta$-actin (forward: 5'-GCACAGAGCCTC GCCTT-3'; reverse: 5'-GTTGTCGACGACGAGCG-3').

Transient transfection of miR-605 mimics or inhibitors. miR-605 mimics or antagomiR-605 transfections were performed with Lipofectamine RNAiMAX Transfection Reagent (Invitrogen) according to the manufacturer's protocols and used for subsequent measurements 24 or $48 \mathrm{~h}$ after transfection.

Cell proliferation assays. Transfected melanoma cells were plated in 96-well plates $(2,000$ cells/well). Cell proliferation was determined using Cell Counting Kit-8 (CCK-8) (Dojindo Laboratories, Tokyo, Japan) according to the manufacturer's instructions. Assays were performed in triplicate, and the results are presented as means \pm standard deviation (SD).

Colony formation assay. Transfected melanoma cells were seeded in a 6 -well plate $(2,000$ cells/well) containing DMEM/5\% FBS medium. Cells were allowed to grow for two weeks and then fixed with methanol and stained with $0.5 \%$ crystal violet solution. The colony numbers were measured using Image J (MD, USA). Assays were performed in triplicate, and the results are presented as means \pm standard deviation (SD).

Soft agar assay. The cells were seed in a layer of $0.35 \%$ agarose containing RPMI-1640/10\% FBS medium at 5,000 cells per well, which was on top of a base layer of $0.5 \%$ agarose in 6-well culture plates. Two weeks after plating, colonies were photographed and counted under a light microscope.

Cloning and luciferase reporter assay. The wild-type or mutant INPP4B 3'-UTR was cloned into pGL3 luciferase reporter plasmid (Promega) following the manufacturer's instructions. All constructs were validated by sequencing.

The cells were plated into 24 -well plates $\left(3 \times 10^{4}\right.$ cell/per well). The following day, pGL3 luciferase reporter plasmid with the wild-type or mutant INPP4B 3'-UTR was co-transfected into melanoma cells along with miR-605 mimics or antagomiR-605 using Lipofectamine 2000 (Invitrogen). The cells were harvested $48 \mathrm{~h}$ post-transfection and luciferase activities were measured with the Dual-Luciferase Reporter Assay system (Promega) according to the manufacturer's suggestions. The pRL-TK vector was used as an internal 
A

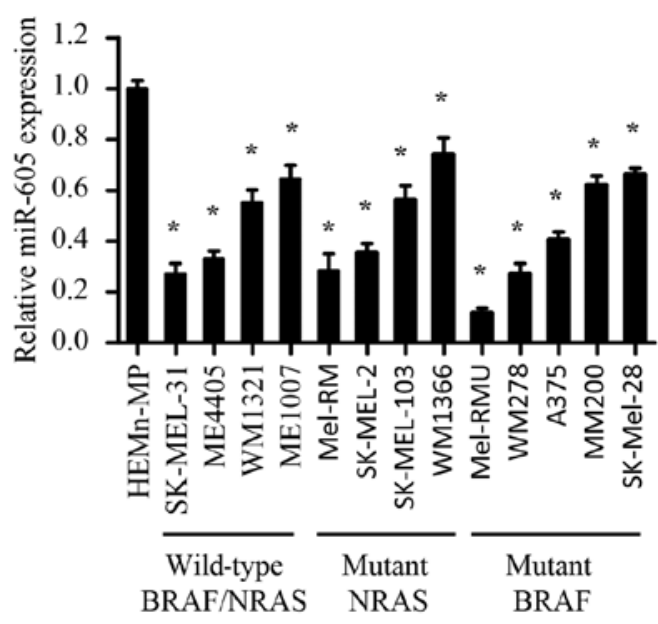

B

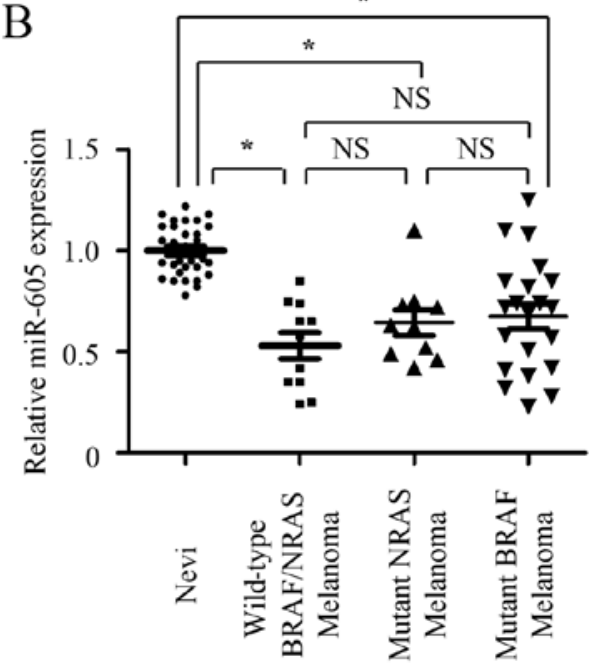

Figure 1. miR-605 is significantly downregulated in melanoma cells and tissues. (A) Relative expression of miR-605 in melanocyte cell line (HEMn-MP), BRAF/NRAS wild-type (SK-MEL-31, ME4405, WM1321 and Me1007), NRAS mutant (Mel-RM, SK-MEL-2, SK-MEL-103 and WM1366) and BRAF mutant (Mel-RMU, WM278, A375, MM200 and SK-Mel-28) melanoma cell lines. The expression of miR-605 was normalized to U6 snRNA (*P<0.05). (B) Comparison of miR-605 expression among nevi $(\mathrm{n}=31)$, BRAF/NRAS wild-type melanomas ( $\mathrm{n}=11)$, NRAS mutant melanomas ( $\mathrm{n}=10)$ and BRAF mutant melanomas $(n=21)$ determined by qRT-PCR. The expression of miR-605 was normalized to U6 snRNA ( $\mathrm{P}<0.05)$.

control. Assays were performed in triplicate and results are presented as means \pm standard deviation $(\mathrm{SD})$.

Western blotting. Western blotting was performed as previously described (32).

In vivo tumorigenesis assay. Five-week-old male BALB/c nude mice were obtained from the Animal Center for Vitalriver (Beijing, China). To measure the role of miR-605 overexpression on the tumor growth, Mel-RM cells stably overexpressing miR-605 or control cells in $0.1 \mathrm{ml}$ OptiMEM were subcutaneously injected into the right flank of nude mice, respectively. To detect the effect of miR-605 inhibition on the tumorigenic ability of melanoma cells, SK-MEL-28 cells were subcutaneously injected into the right flank of nude mice. When the tumor reached an average volume of $100 \mathrm{~mm}^{3}$, the nude mice bearing tumor were randomly divided to 2 groups ( $n=6 /$ group) according to tumor volumes and body weights and received intratumoral injection of antagomir-605 $(10 \mathrm{nM}$ of antagomir-605 diluted in $50 \mu \mathrm{l}$ PBS) or antagomir-NC for 3 weeks (three times per week). Tumor growth were monitored at indicated times.

Statistical analysis. Statistical analysis was performed with the unpaired Student's t-test using the SPSS 17.0 software (SPSS, Chicago, IL, USA). The data are presented as mean \pm standard deviation (SD). P-values of $<0.05$ were considered statistically significant.

\section{Results}

miR-605 is significantly downregulated in melanoma cells and tissues. To explore the significance of miR-605 in the initiation and progression of melanoma, we first determined the expression of miR-605 in a panel of BRAF/NRAS wildtype (SK-MEL-31, ME4405, WM1321 and Me1007), NRAS mutant (Mel-RM, SK-MEL-2, SK-MEL-103 and WM1366) and BRAF mutant (Mel-RMU, WM278, A375, MM200 and SK-Mel-28) melanoma cell lines. The results show that miR-605 exhibits various expression levels in three types of melanoma cell lines. Although miR-605 expression was significantly lower in melanoma cell lines compared to melanocyte cell line, there was no significant difference in the three types of melanoma cell lines (Fig. 1A).

Next, we investigated whether miR-605 is downregulated in clinical melanoma samples. The abundance of miR-605 was examined in 73 clinical samples, including nevi $(n=31)$, nevi $(n=31)$, BRAF/NRAS wild-type melanomas $(n=11)$, NRAS mutant melanomas $(n=10)$ and BRAF mutant melanomas $(n=21)$. In agree with the results in cell lines, the expression of miR-605 in three types of melanomas was markedly decreased compared with that in nevi. However, miR-605 expression exhibited no statistical difference in three types of melanomas clinical samples (Fig. 1B). Together, our data demonstrate that miR-605 is significantly downregulated in melanoma cells and tissues but shows no correlation with the mutational status of melanoma cell lines or the clinical specimens, suggesting the miR-605 may play a potential role in the initiation and progression of melanomas. Furthermore, we examined the expression levels of miR-605 in 73 clinical samples.

Upregulation of miR-605 suppresses the growth of melanoma cells. The reduced expression of miR-605 in melanomas indicates that it may function as tumor suppressor. To access the role of miR-605, the miR-605 mimics were transfected into Mel-RM and ME4405 cells, which expressed relatively low levels of miR-605 (Fig. 1A). As shown in Fig. 2A, the expression of miR-605 in Mel-RM and ME4405 cells was significantly increased by miR-605 mimics transfection. CCK8 assays showed that ectopic expression of miR-605 resulted in a significant inhibition in the growth of both Mel-RM and ME4405 cells (Fig. 2B). Furthermore, the clonogenic potential 

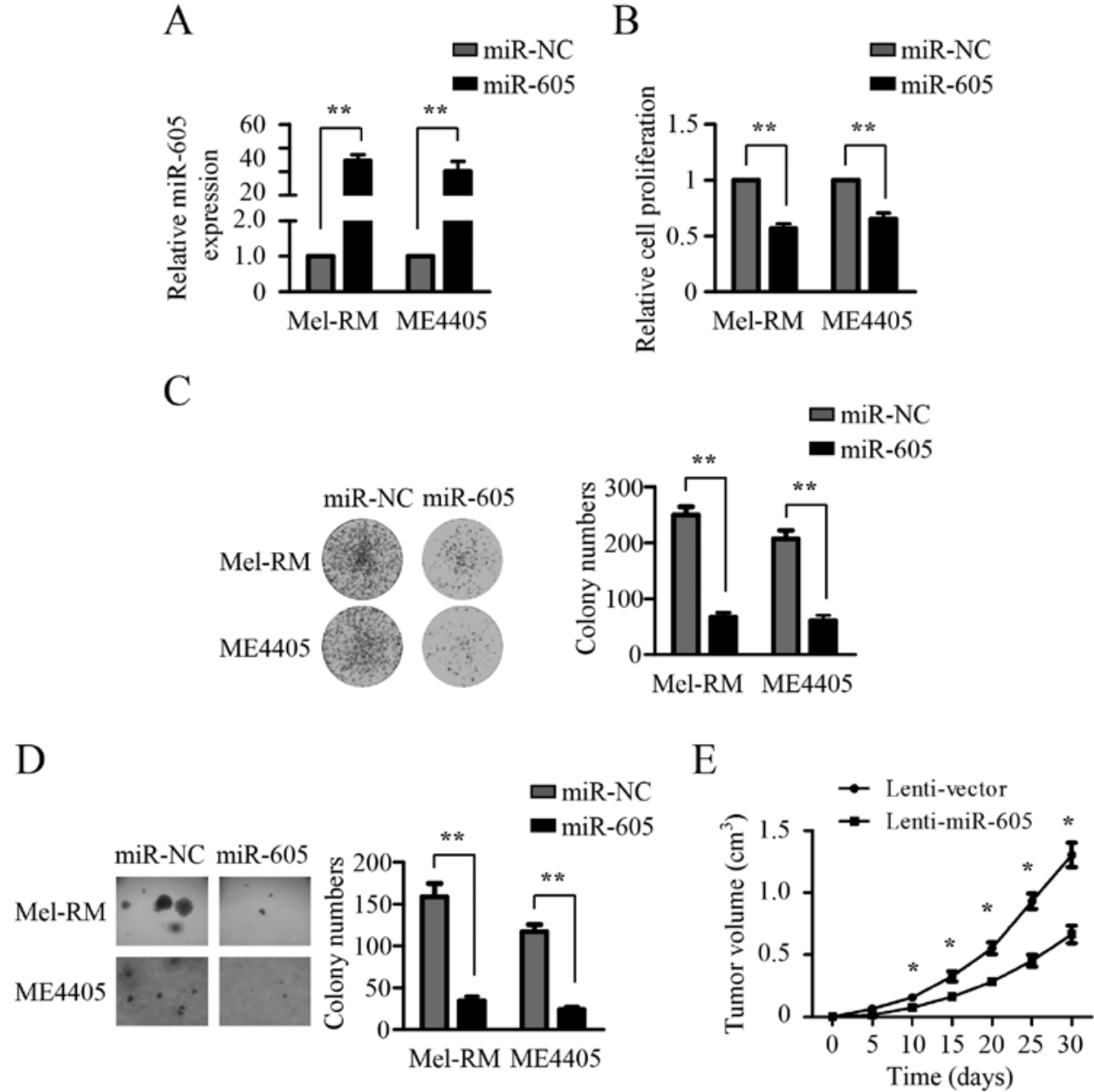

Figure 2. Upregulation of miR-605 suppresses the growth of melanoma cells. (A) miR-NC or miR-605 was transfected into Mel-RM and ME4405 cells. Relative expression of miR-605 was accessed by RT-PCR after $48 \mathrm{~h}$ of transfection (n=3). Mel-RM and ME4405 cells were transfected with miR-NC or miR-605. Twenty-four hours later, cells were subjected to CCK-8 assay (B), colony formation assay (C) and soft agar assay (D). Results are shown as means \pm SD of triplicate measurements. ${ }^{*} \mathrm{P}<0.05$. (E) Comparison of growth curves of xenografts of stably overexpressing miR-605 and control cells). The data shown are mean \pm SEM, $n=6 .{ }^{*} \mathrm{P}<0.05 ;{ }^{* *} \mathrm{P}<0.01$.

of Mel-RM and ME4405 cells was markedly suppressed upon miR-605 overexpression (Fig. 2C). Soft agar assays demonstrated that Mel-RM and ME4405 cells expressing miR-605 both exhibited substantially decreased anchorage-independent growth ability (Fig. 2D). These observations indicated that enforced expression of miR-605 inhibited the growth of melanomas cells in vitro, and we next detected the effect of miR-605 overexpression on the growth of melanoma cells in vivo. Mel-RM cells stably overexpressing miR-605 were established and injected into the flanks of nude mice. As shown in Fig. 2E, miR-605 overexpression profoundly suppressed the growth of subcutaneous xenograft tumors. Taken together, these findings suggest that miR-605 has a suppressor role in the growth of melanomas cells.

Inhibition of miR-605 promotes the growth of melanoma cells. To confirm the inhibitory role of miR-605 in the growth of melanoma cells, we next evaluated the impact of miR-605 inhibition on the growth of melanoma cells. To silence the expression of miR-605, Antagomir-605 was transfected into MM200 and SK-MEL 28 cells, which showed relatively higher expression levels of miR-605 compared with Mel-RM and ME4405 cells (Fig. 1A). As shown in Fig. 3A, antago-miR-605 transfection profoundly reduced endogenous miR-605 expression in both MM200 and SK-MEL 28 cells. CCK8 assays revealed that inhibition of miR-605 significantly promoted the growth of both MM200 and SK-MEL 28 cells (Fig. 3B). Colony formation assays demonstrated that MM200 and SK-MEL 28 cells transfected with antagomir-605 showed a marked increase in both the size and number of colonies compared to the cells transfected with antagomir-NC (Fig. 3C). Soft agar assays showed that miR-605 depletion strikingly promoted anchorage-independent growth ability of both MM200 and SK-MEL 28 cells (Fig. 3D). To validate the in vitro finding that silencing the expression of miR-605 enhanced the growth of melanoma cells, we subsequently determined the effect of miR-605 inhibition on the tumorigenic ability of melanoma cells. SK-MEL-28 cells were injected subcutaneously into nude mice. After two weeks, nude mice bearing tumors were randomly divided to 2 groups and injected with antagomir-605 or negative control for three weeks. As shown in Fig. 3E, antagomir-605 treatment profoundly increased tumorigenic ability of SK-MEL 28 cells in vivo. These results suggest that inhibition of miR-605 promotes the growth of melanoma cells in vitro and in vivo. 
A

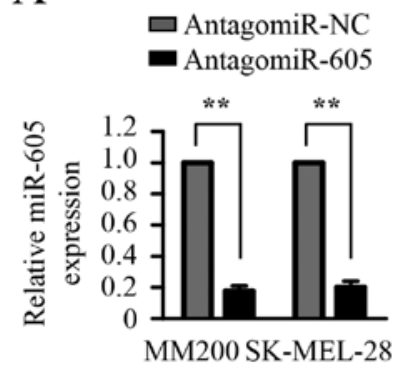

C

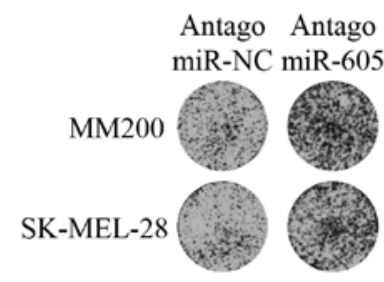

B
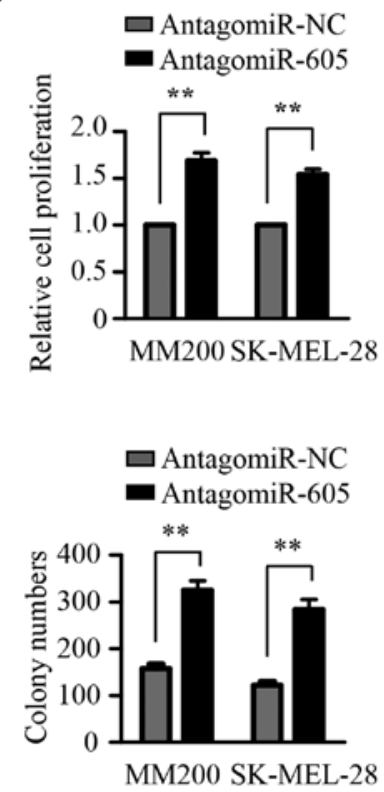

D

E
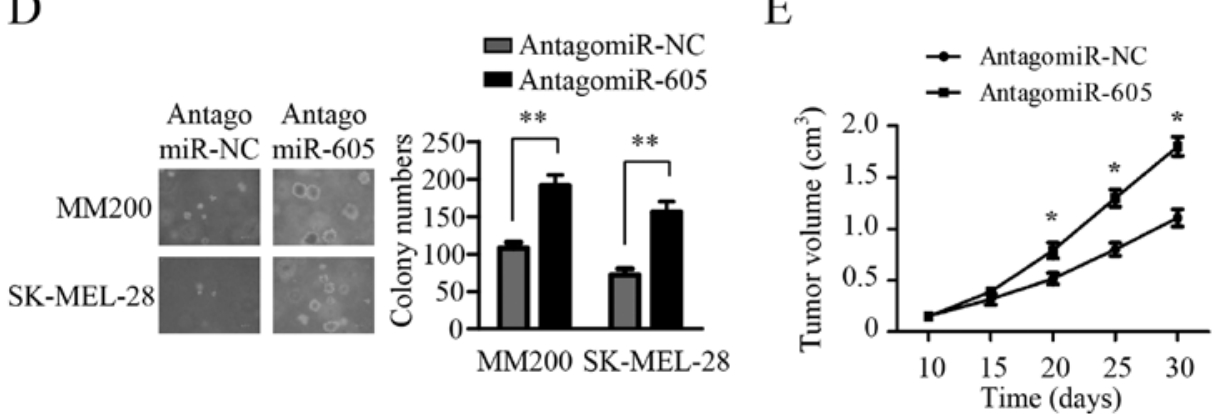

Figure 3. Inhibition of miR-605 promotes the growth of melanoma cells. (A) Relative miR-605 levels in MM200 and SK-MEL-28 cells following 48 h transfection with antagomir-NC or antagomir-605 (n=3). MM200 and SK-MEL-28 cells were transfected with antagomir-NC or antagomir-605, followed by CCK-8 assay (B) colony formation assay (C) and soft agar assay (D) twenty-four hours after transfection. Results are shown as means \pm SD of triplicate measurements. ${ }^{*} \mathrm{P}<0.05$. (E) SK-MEL-28 cells were injected subcutaneously in the right flank of nude mice. When the average tumor volume had reached approximately $100 \mathrm{~mm}^{3}$, the mice were randomly divided to 2 groups (n=6/group) and intratumorally injected antagomir-NC or antagomir-605 once every two days for 20 days. Tumor growth was monitored at the indicated times. ${ }^{*} \mathrm{P}<0.05 ;{ }^{* *} \mathrm{P}<0.01$.

INPP4B is a direct target of miR-605. To investigate the molecular mechanisms by which miR-605 inhibits the growth of melanomas cells, we searched for its target genes using two bioinformatics tools, miRanda and TargetScan. INPP4B, one of putative target genes of miR-605, attracted our attention due to its oncogenic activity in melanomas reported recently and one possible binding site of miR-605 in its 3'-untranslated region (3'-UTR) (Fig. 4A). To determine whether miR-605 targets INPP4B, we cloned the 3'-UTR of wild-type and mutant INPP4B (mutations in miR-605 binding sites) into a luciferase reporter plasmid and performed the luciferase activity assay. As shown in Fig. 4B, miR-605 transfection significantly suppressed luciferase activity in both Mel-RM and ME4405 cells, whereas this inhibitory effects was abolished by the mutation of the potential miR-605 binding sequence in the 3'-UTRs of INPP4B, suggesting that INPP4B is a direct target of miR-605. The opposite result was obtained in MM200 and SK-MEL-28 cells transfected with antagomir-605 (Fig. 4C), revealing that the 3 '-UTRs of INPP4B was inhibited by endogenous miR-605.
In addition, western blot showed that ectopic expression of miR-605 suppressed its protein expression in Mel-RM and ME4405 cells, whereas inhibition of endogenous miR-605 increased the expression of INPP4B in MM200 and SK-MEL-28 cells (Fig. 4F and G). However, miR-605 expression levels did not alter the mRNA expression of INPP4B (Fig. 4D and E), suggesting that miR-605 negatively regulates INPP4B expression by repressing its mRNA translation, but not enhancing its mRNA degradation. Taken together, these results suggest that miR-605 negatively regulates the expression of INPP4B through directly targeting its 3'-UTR. To explore the correlation between miR-605 and INPP4B levels in clinical specimens, we collected 10 pairs of nevi and melanomas tissues from the same patients and examined miR-605 and INPP4B protein expression. As shown in Fig. $4 \mathrm{H}$, when the relative expression levels of INPP4B (melanomas/nevi) were plotted against that of miR-605 (melanomas/nevi) in each patient, a significant inverse correlation was found $(\mathrm{P}<0.0322 ; \mathrm{r}=-0.675)$. These data indicate that miR-605 downregulation is associated with the increase of INPP4B protein levels in melanomas. 
A

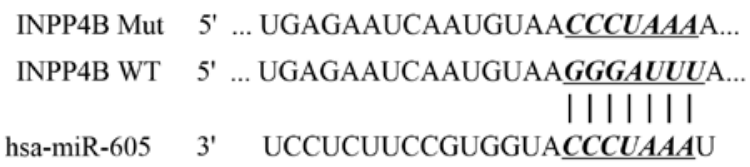

B

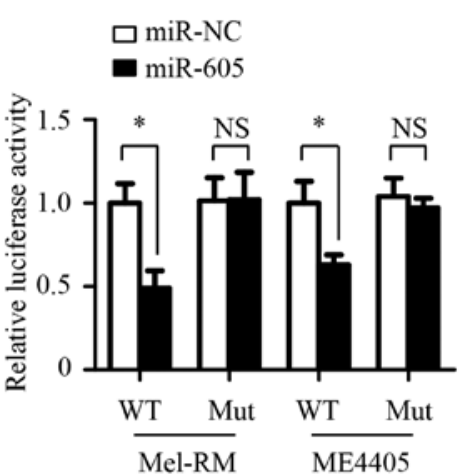

$\mathrm{D}$

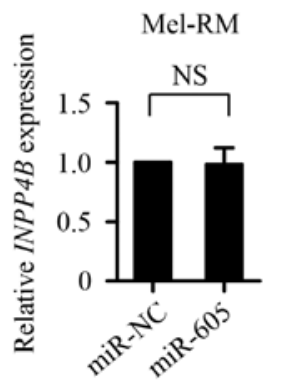

$\mathrm{F}$

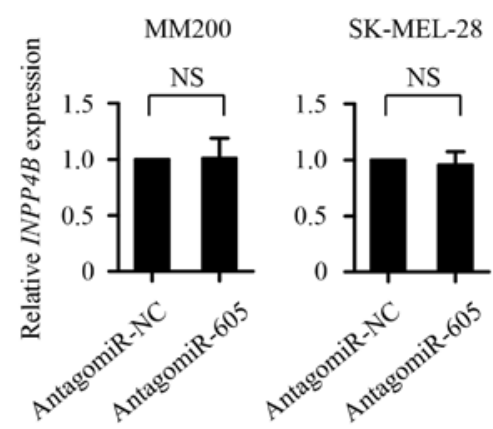

$\mathrm{C}$

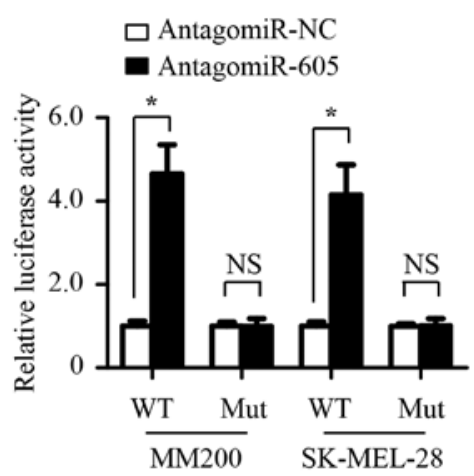

E

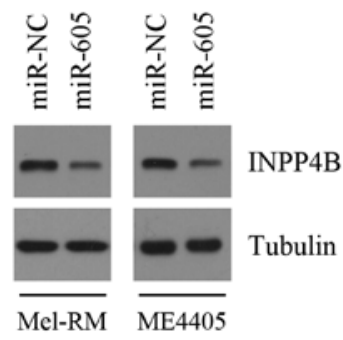

G

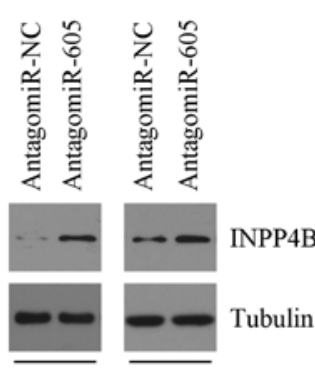

MM200 SK-MEL-28

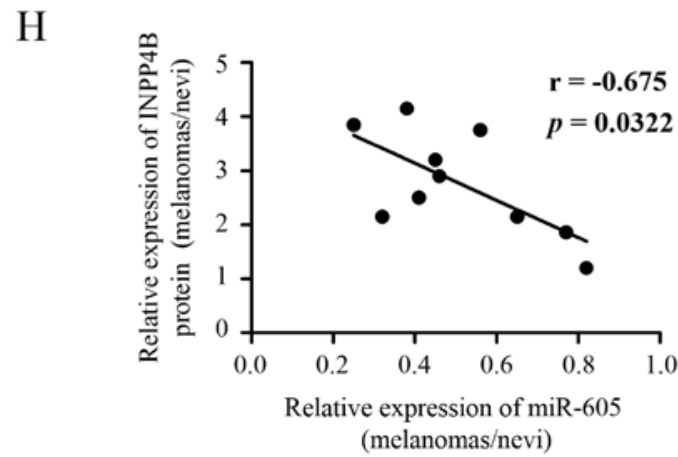

Figure 4. miR-605 directly targets INPP4B. (A) Scheme of the target sites of miR-605 in 3'-UTR of INPP4B mRNA. (B) Wild-type or mutant INPP4B 3'-UTR luciferase reporter vector in combination with miR-NC or miR-605 were co-transfected into Mel-RM and ME4405 cells. Luciferase activities were detected after $48 \mathrm{~h}$. Results are shown as means \pm SD of triplicate measurements. "P<0.05; NS, not significant. (C) Wild-type or mutant INPP4B 3'-UTR luciferase reporter vector was transfected into MM200 and SK-MEL-28 cells along with either antagomir-NC or antagomir-605. Forty-eight hours later, luciferase activities were monitored. Results are shown as means $\pm \mathrm{SD}$ of triplicate measurements. $\mathrm{P}<0.05$; NS, not significant. qRT-PCR analysis of INPP4B mRNA levels in Mel-RM and ME4405 cells transfected with miR-NC or miR-605 (D) and MM200 and SK-MEL-28 cells transfected with antagomir-NC or antagomir-605 (F). Expression of INPP4B mRNA was normalized to actin. Results are shown as means \pm SD of triplicate measurements. NS, not significant. The INPP4B protein levels were detected by western blot analysis in Mel-RM and ME4405 cells transfected with miR-NC or miR-605 (E) and MM200 and SK-MEL-28 cells transfected with antagomir-NC or antagomir-605 (G). Tubulin was used as a loading control. (H) Inverse correlation between miR-605 expression and INPP4B protein in melanomas versus nevi from the same patient. A statistically significant correlation between miR-605 expression and INPP4B protein was observed by Pearson's method with a correlation coefficient of -0.675 . 
A

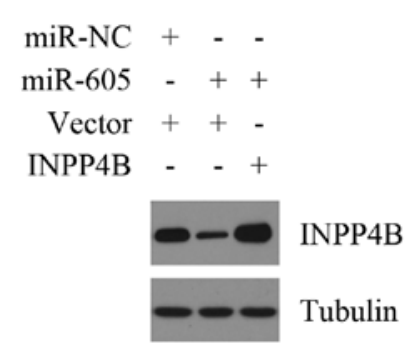

B

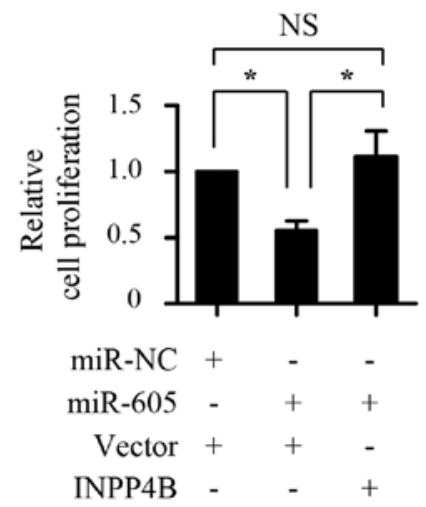

C
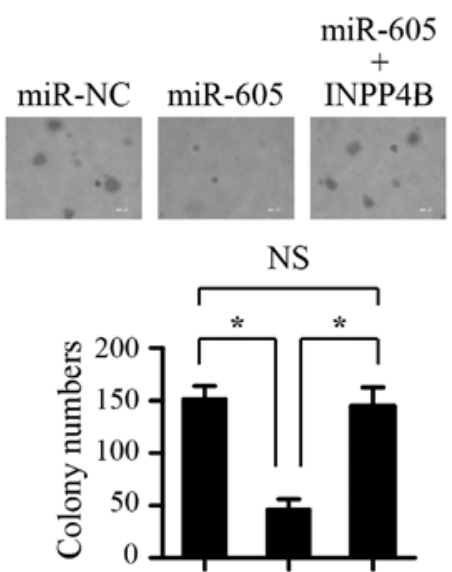

miR-NC + - -

miR-605 - + +

Vector + + -

INPP4B - $\quad$ +
D

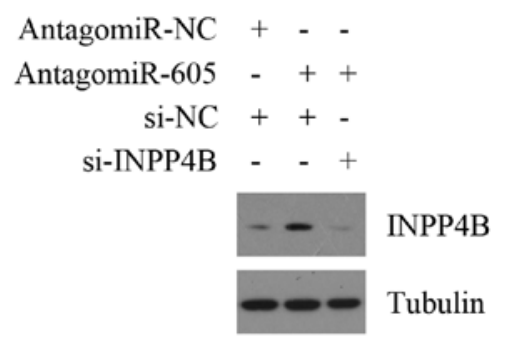

E

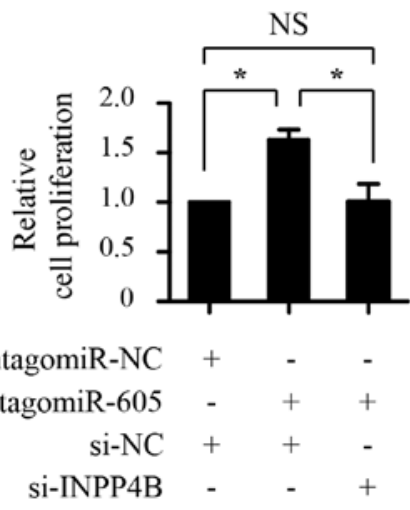

F
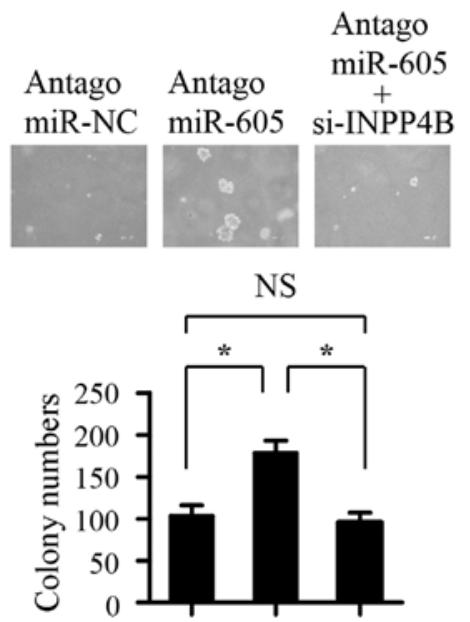

AntagomiR-NC + - -

AntagomiR-605 - + +

si-NC + + -

si-INPP4B - $\quad$ - $\quad+$

Figure 5. miR-605 suppresses the growth of melanoma cells by inhibiting INPP4B. (A) Western blot analysis of INPP4B expression in Mel-RM cells co-transfected with miR-NC + pcDNA3.1 vector, miR-605 + pcDNA3.1 vector or miR-605 + pcDNA3.1-INPP4B. Tubulin was used as a loading control. CCK8 assay (B) and soft agar assay (C) of Mel-RM cells in indicated groups. The means \pm SD of triplicate measurements are shown. "P<0.05; NS, not significant. (D) MM200 cells were co-transfected with antagomir-NC or antagomir-605 and the control or INPP4B siRNA and were subjected to western blot analysis. Tubulin was used as a loading control. CCK8 assay (E) and soft agar assay (F) of MM200 cells transfected with antagomir-NC or antagomir-605 and the control or INPP4B siRNA. The means \pm SD of triplicate measurements are shown. ${ }^{*} \mathrm{P}<0.05$; NS, not significant.

miR-605 suppresses the growth of melanoma cells by inhibiting INPP4B. To investigate the functional significance of INPP4B in the growth of melanomas cells suppressed by miR-605, 3' UTR-deleted INPP4B plasmid was introduced into Mel-RM cells transfected with miR-605 mimics, and then cell proliferation and anchorage-independent growth ability of melanomas cells were measured by CCK8 assays and soft agar growth assay, respectively. As shown in Fig. 5A-C, miR-605 mimics transfection suppressed proliferation of Mel-RM cells and resulted in decreased anchorage-independent growth ability, whereas introduction with INPP4B plasmid rescued the phenotypic alteration caused by miR- 605 overexpression. To confirm that INPP4B is a functional target of miR-605, we next detected the impact of INPP4B silencing on antagomir-605-mediated promotion of proliferation and anchorage-independent growth ability of melanomas 
A

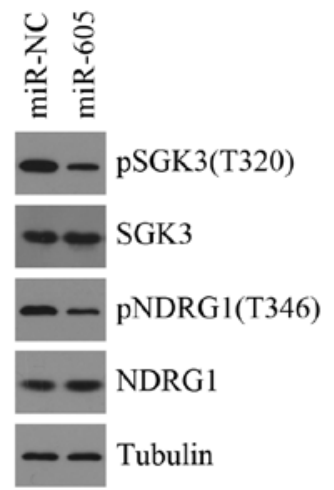

$\mathrm{D}$

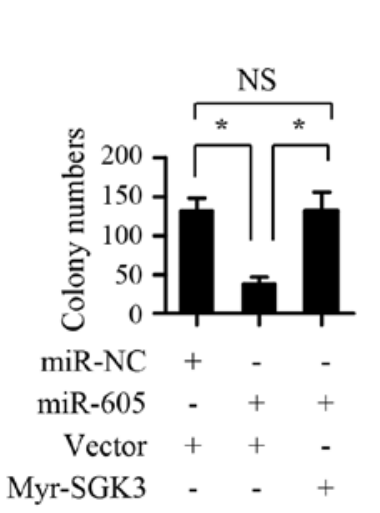

B

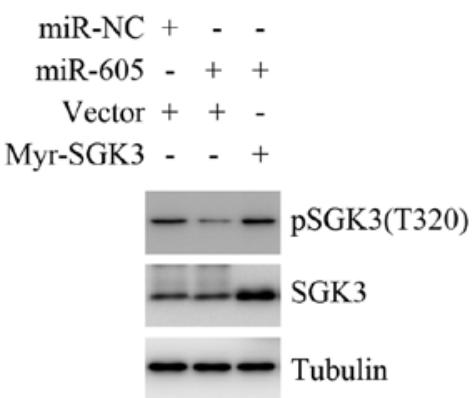

E

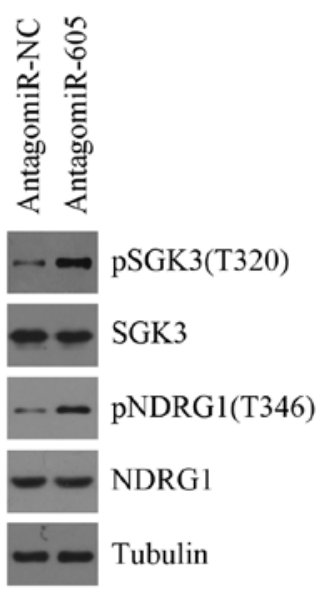

C

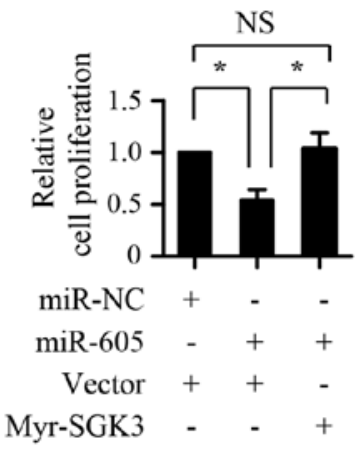

G

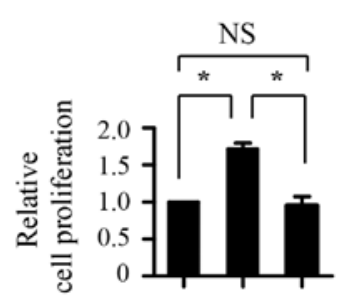

AntagomiR-NC + - -

AntagomiR-605 - + +

si-NC + + -

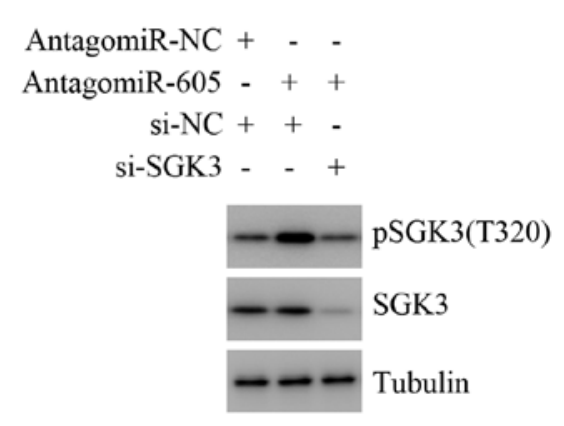

$\mathrm{H}$

\section{F}

si-SGK3

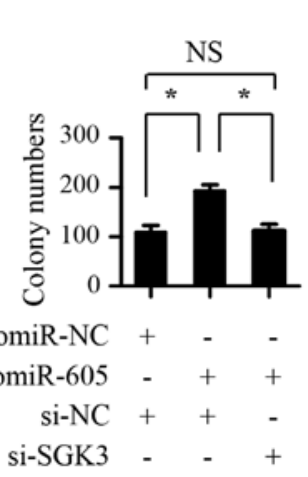

Figure 6. The inhibition of SGK3 activity is crucial for the suppressive role of miR-605 on melanomas cell growth. (A) Mel-RM cells were transfected with miR-NC or miR-605. Cell lysates were harvested after $48 \mathrm{~h}$ and were subjected to western blot analysis with the indicated antibodies. Mel-RM cells were co-transfected with miR-NC + pcDNA3.1 vector, miR-605 + pcDNA3.1 vector or miR-605 + pcDNA3.1-Myr-SGK3 and were subjected to western blot analysis (B), CCK8 assay (C) and soft agar assay (D). The means \pm SD of triplicate measurements are shown. "P<0.05; NS, not significant. (E) MM200 cells transfected with antagomir-NC or antagomir-605 were used for western blot analysis with the indicated antibodies. Tubulin was used as a loading control. Western blot analysis (F), CCK8 assay $(\mathrm{G})$ and soft agar assay $(\mathrm{H})$ of MM200 cells in indicated groups. The means \pm SD of triplicate measurements are shown. ${ }^{*} \mathrm{P}<0.05$; NS, not significant.

cells. As shown in Fig. 5D-F, MM200 cells transfected with antagomir-605 exhibited markedly increased cell proliferation and anchorage-independent growth ability, whereas INPP4B knockdown abrogated the increase. Taken together, these results suggest that miR-605 suppresses the growth of melanomas cells by inhibiting INPP4B.

The inhibition of SGK3 activity is crucial for the suppressive role of miR-605 on melanoma cell growth. Given that one recent study demonstrated that SGK3 activation was critical for INPP4B-mediated melanoma cell proliferation (13) and the above results revealed that INPP4B is a functional target of miR-605, we presumed that the inhibition of SGK3 activity may mediate the suppressive role of miR-605 on melanomas cell growth. To test this hypothesis, we first detected the effect of miR-605 expression levels on the activity of SGK3. As shown in Fig. 6A, miR-605 overexpression in Mel-RM cells inhibited the phosphorylation levels of SGK3 as well as NDRG1, one downstream substrate of SGK3. In contrast, silencing the expression endogenous miR-605 using antagomir-605 
significantly enhanced the phosphorylation levels of SGK3 and NDRG1 in MM200 cells (Fig. 6E), indicating that miR-605 inhibits the activity of SGK3 in melanoma cells. We next explored whether the inhibition of SGK3 activity contributes to the suppressive role of miR-605 on melanoma cell growth. As shown in Fig. 6B-D, introduction of miR-605 mimics led to inhibition of proliferation and anchorage-independent growth ability in Mel-RM cells, which was abolished by co-introduction of exogenous myr-SGK3. Furthermore, the increase of proliferation and anchorage-independent growth ability in Mel-RM cells caused by miR-605 inhibition could be markedly attenuated by SGK3 depletion (Fig. 6F and G). Taken together, these results suggest that the inhibition of SGK3 activity is crucial for the suppressive role of miR-605 on melanoma cell growth.

\section{Discussion}

Increasing evidence demonstrates that miRNAs display altered expression levels in a variety of cancer types and play a key role in the initiation and progression of cancer. Therefore, miRNAs have been extensively investigated to identify novel biomarkers for cancer diagnosis and prognosis and develop effective therapeutic strategy to treat cancer patients. In melanoma, many miRNAs exhibit abnormal expression and are involved in tumor progression. miR-182 was reported to be upregulated in melanoma cell lines and tissues and its expression levels were associated with melanoma progression and malignancy. Moreover, miR-182 contributed to melanoma development through directly targeting FOXO3 (22). miR-137 was the first identified tumor suppressor in melanoma. miR-137 was downregulated in melanoma and its reduced expression correlated with reduced overall survival in stage IV melanoma patients. miR-137 exerts tumor suppressor role by targeting multiple oncogenes, including c-Met, EZH2, PAK2 and AURKA $(25,33,34)$.

miR-605 was originally identified as a positive regulator of P53 through post-transcriptionally repressing the expression of $\mathrm{Mdm} 2$, which facilitates rapid accumulation of p53 in response to cellular stress (27). The studies of miR-605 in cancer mainly focus on the association of the genetic variants in miR-605 with cancer susceptibility. Chen et al reported a decreased risk of breast cancer in miR-605 rs2043556*A allele carriers in Asia (35). It was shown that AG and GG genotype carriers of miR-605 rs2043556 who has exposure to cooking oil fumes displayed an increased risk of lung cancer compared with AA genotype carriers without exposure to cooking oil fumes (30). Zhang et al reported that miR-605 AG/GG genotype carriers with the habit of smoke inhalation predicted elevated risk of gastric cancer (28). Recent studies also revealed the abnormal expression of miR-605 in cancers. miR-605 showed a significantly decreased expression in very high-risk (VHR) prostate cancer patient serum samples when compared with low-risk (LR) prostate cancer patient serum samples (36). It was reported that miR-605 showed a decreased expression in intrahepatic cholangiocarcinoma (ICC) specimens and suppressed ICC cell proliferation and invasion by directly targeting PSMD10 (31). However, the expression status of the miR-605 in melanoma tissues and its role in melanoma progression are unclear. In this study, we found that miR-605 showed decreased expression level in melanomas when compared with nevi, suggesting that miR-605 is associated with melanomagenesis and it may act as a tumor suppressor. Further studies revealed that miR-605 inhibited anchorage-dependent and -independent growth of melanoma cells. In addition, in vivo studies demonstrated that miR-605 overexpression caused retardation in melanoma growth in a xenograft model. All these studies indicate that miR-605 exhibits tumor suppressor role in melanomagenesis.

Through in silico algorithms analyses, we identified INPP4B as a putative target. Reporter assays demonstrated that miR-605 inhibited the expression of INPP4B by directly binding to its 3'-UTR. Further analysis showed that miR-605 suppressed the protein expression level of INPP4B, but had little effect on its mRNA expression level, suggesting that miR-605 negatively regulates INPP4B expression by repressing its mRNA translation. Although numerous studies demonstrate that INPP4B acts as a tumor suppressor through inhibition of $\mathrm{PI} 3 \mathrm{~K} / \mathrm{Akt}$ signaling in many types of cancers, recent reports show its oncogenic role in some cancers. INPP4B showed markedly elevated expression in colon cancer tissues when compared with paired adjacent noncancerous colon tissues and its overexpression significantly promoted colon cancer cell proliferation and colon cancer xenograft growth (37). High levels of INPP4B were observed in acute myeloid leukemia patient samples and predicted poor clinical outcome (10). A recent study pointed to INPP4B as an essential effector of oncogenic PIK3CA activated breast cancer (12). In particular, INPP4B was recently reported to be highly expressed in melanoma and to promote proliferation of melanoma cells (13), suggesting an oncogenic role of INPP4B in melanoma. Our results showed that enforced expression of INPP4B effectively reversed reduced cell proliferation and anchorage-independent growth caused by miR-605 overexpression, indicating the functional significance of INPP4B in mediating the tumor suppresser role of miR-605 and further confirming the oncogenic role of INPP4B in melanoma. Whether miR-605 and INPP4B show the reciprocal expression in clinical melanoma tissues remains to be clarified.

The activation of SGK3 is controlled by cellular PtdIns(3)P, which binds to the N-terminal PX domain of SGK3 and mediates its translocation to early endosomes for phosphorylation by its upstream PDK1 kinase (14). Therefore, INPP4B mediated PtdIns(3)P generation may contribute to the activation of SGK3. SGK3 has been reported to mediate the oncogenic role of INPP4B in many cancers, including lung cancer, breast cancer and melanoma $(6,12,13)$. In either melanoma cell lines or fresh melanoma isolates with various levels of INPP4B, the phosphorylation levels of SGK3 are positively correlated with the expressing levels of INPP4B. INPP4B depletion significantly inhibited the phosphorylation levels of SGK3 and cell proliferation in melanoma cells and introduction of an active form of SGK3 (myr-SGK3) can abolished the suppressive effect of INPP4B knockdown on melanoma cell proliferation, revealing SGK3 activation is essential for INPP4B-induced melanoma cell proliferation. As we have validated that miR-605 suppresses the growth of melanoma cells by inhibiting INPP4B, we speculated that miR-605 might affect the activation of SGK3. Our results showed that miR-605 overexpression profoundly inhibited the phosphorylation levels of SGK3 and its target NDRG1, which verified our hypothesis. 
Further studies demonstrate that co-introduction of exogenous myr-SGK3 markedly attenuated suppression of proliferation resulting from miR-605 overexpression, suggesting that the inhibition of SGK3 activity is required for miR-605-mediated melanoma cell growth suppression. A recent report showed that SGK3 could activating mTORC1 signaling through phosphorylating TSC2 (38). As mTORC1 signaling plays a critical role in the initiation and progression of melanoma and contributes to the development of resistance to BRAF inhibitors (39), it would be of interest to investigate the association of miR-605 repression with mTORC1 signaling activation and the resistance to anti-BRAF therapies in melanoma.

In summary, in the present study we present evidence that miR-605 functions as a tumor suppressor by repressing INPP4B expression and SGK3 activity in melanoma progression. miR-605 was significantly downregulated in melanoma cells and tissues and suppressed the growth of melanoma cells in vitro and in vivo. Although additional studies are required to address the mechanism involved in the reduced expression of miR-605, our results indicate that miR-605 provide novel insight into molecular basis regulating melanoma malignancy and are helpful to develop novel therapeutic approach to treat melanoma patients.

\section{Acknowledgements}

This study was supported by Guizhou Province Chinese Native Medicine Modernization Special Project (20125018 to Y.C.) and Guiyang Science and Technology Bureau Science and Technology Innovation Platform Project (2012303 to Y.C.).

\section{References}

1. Arrangoiz R, Dorantes J, Cordera F, Juarez MM and Paquentin EM: Melanoma review: Epidemiology, risk factors, diagnosis and staging. J Cancer Treat Res 4: 1-15, 2016. doi: $10.11648 /$ j.jctr.20160401.11.

2. Siegel RL, Miller KD and Jemal A: Cancer statistics, 2016. CA Cancer J Clin 66: 7-30, 2016.

3. Chen W: Cancer statistics: updated cancer burden in China. Chin J Cancer Res 27: 1, 2015.

4. Holderfield M, Deuker MM, McCormick F and McMahon M Targeting RAF kinases for cancer therapy: BRAF-mutated melanoma and beyond. Nat Rev Cancer 14: 455-467, 2014.

5. Agoulnik IU, Hodgson MC, Bowden WA and Ittmann MM: INPP4B: The new kid on the PI3K block. Oncotarget 2: 321-328, 2011.

6. Zhang L, Zeng D, Chen Y, Li N, Lv Y, Li Y, Xu X and Xu G: miR-937 contributes to the lung cancer cell proliferation by targeting INPP4B. Life Sci 155: 110-115, 2016.

7. Rynkiewicz NK, Fedele CG, Chiam K, Gupta R, Kench JG, Ooms LM, McLean CA, Giles GG, Horvath LG and Mitchell CA: INPP4B is highly expressed in prostate intermediate cells and its loss of expression in prostate carcinoma predicts for recurrence and poor long term survival. Prostate 75: 92-102, 2015.

8. Hsu I, Yeh CR, Slavin S, Miyamoto H, Netto GJ, Tsai YC, Muyan M, Wu XR, Messing EM, Guancial EA, et al: Estrogen receptor alpha prevents bladder cancer via INPP4B inhibited akt pathway in vitro and in vivo. Oncotarget 5: 7917-7935, 2014.

9. Perez-Lorenzo R, Gill KZ, Shen CH, Zhao FX, Zheng B, Schulze HJ, Silvers DN, Brunner G and Horst BA: A tumor suppressor function for the lipid phosphatase INPP4B in melanocytic neoplasms. J Invest Dermatol 134: 1359-1368, 2014.

10. Dzneladze I, He R, Woolley JF, Son MH, Sharobim MH, Greenberg SA, Gabra M, Langlois C, Rashid A, Hakem A, et al: INPP4B overexpression is associated with poor clinical outcome and therapy resistance in acute myeloid leukemia. Leukemia 29: $1485-1495,2015$
11. Fedele CG, Ooms LM,Ho M, Vieusseux J, O'Toole SA, MillarEK Lopez-Knowles E, Sriratana A, Gurung R, Baglietto L, et al: Inositol polyphosphate 4-phosphatase II regulates PI3K/Akt signaling and is lost in human basal-like breast cancers. Proc Natl Acad Sci USA 107: 22231-22236, 2010.

12. Gasser JA, Inuzuka H, Lau AW, Wei W, Beroukhim R and Toker A: SGK3 mediates INPP4B-dependent PI3K signaling in breast cancer. Mol Cell 56: 595-607, 2014.

13. Chi MN, Guo ST, Wilmott JS, Guo XY, Yan XG, Wang CY, Liu XY, Jin L, Tseng HY, Liu T, et al: INPP4B is upregulated and functions as an oncogenic driver through SGK3 in a subset of melanomas. Oncotarget 6: 39891-39907, 2015.

14. Bruhn MA, Pearson RB, Hannan RD and Sheppard KE: AKT-independent PI3-K signaling in cancer - emerging role for SGK3. Cancer Manag Res 5: 281-292, 2013.

15. Wang Y, Zhou D, Phung S, Masri S, Smith D and Chen S: SGK3 is an estrogen-inducible kinase promoting estrogen-mediated survival of breast cancer cells. Mol Endocrinol 25: 72-82, 2011.

16. Wang Y, Zhou D and Chen S: SGK3 is an androgen-inducible kinase promoting prostate cancer cell proliferation through activation of p70 S6 kinase and up-regulation of cyclin D1. Mol Endocrinol 28: 935-948, 2014.

17. Liu H, Li C, Shen C, Yin F, Wang K, Liu Y, Zheng B, Zhang W, Hou X, Chen X, et al: MiR-212-3p inhibits glioblastoma cell proliferation by targeting SGK3. J Neurooncol 122: 431-439, 2015.

18. Liu M, Chen L, Chan TH, Wang J, Li Y, Li Y, Zeng TT, Yuan YF and Guan XY: Serum and glucocorticoid kinase 3 at $8 \mathrm{q} 13.1$ promotes cell proliferation and survival in hepatocellular carcinoma. Hepatology 55: 1754-1765, 2012.

19. Scortegagna M, Lau E, Zhang T, Feng Y, Sereduk C, Yin H, De SK, Meeth K, Platt JT, Langdon CG, et al: PDK1 and SGK3 contribute to the growth of BRAF-mutant melanomas and are potential therapeutic targets. Cancer Res 75: 1399-1412, 2015.

20. Di Leva G, Garofalo M and Croce CM: MicroRNAs in cancer. Annu Rev Pathol 9: 287-314, 2014.

21. Haflidadóttir BS, Bergsteinsdóttir K, Praetorius C and Steingrímsson E: miR-148 regulates Mitf in melanoma cells. PLoS One 5: e11574, 2010.

22. Segura MF, Hanniford D, Menendez S, Reavie L, Zou X, Alvarez-Diaz S, Zakrzewski J, Blochin E, Rose A, Bogunovic D, et al: Aberrant miR-182 expression promotes melanoma metastasis by repressing FOXO3 and microphthalmia-associated transcription factor. Proc Natl Acad Sci USA 106: 1814-1819, 2009.

23. Felicetti F, Errico MC, Bottero L, Segnalini P, Stoppacciaro A, Biffoni M, Felli N, Mattia G, Petrini M, Colombo MP, et al: The promyelocytic leukemia zinc finger-microRNA-221/-222 pathway controls melanoma progression through multiple oncogenic mechanisms. Cancer Res 68: 2745-2754, 2008.

24. Luo C, Merz PR, Chen Y, Dickes E, Pscherer A, Schadendorf D and Eichmüller SB: MiR-101 inhibits melanoma cell invasion and proliferation by targeting MITF and EZH2. Cancer Lett 341: 240-247, 2013.

25. Luo C, Tetteh PW, Merz PR, Dickes E, Abukiwan A, HotzWagenblatt A, Holland-Cunz S, Sinnberg T, Schittek B, Schadendorf D, et al: miR-137 inhibits the invasion of melanoma cells through downregulation of multiple oncogenic target genes. J Invest Dermatol 133: 768-775, 2013.

26. Liu S, Tetzlaff MT, Cui R and Xu X: miR-200c inhibits melanoma progression and drug resistance through down-regulation of BMI-1. Am J Pathol 181: 1823-1835, 2012.

27. Xiao J, Lin H, Luo X, Luo X and Wang Z: miR-605 joins p53 network to form a p53:miR-605:Mdm2 positive feedback loop in response to stress. EMBO J 30: 524-532, 2011.

28. Zhang MW, Jin MJ, Yu YX, Zhang SC, Liu B, Jiang X, Pan YF Li QI, Ma SY and Chen K: Associations of lifestyle-related factors, hsa-miR-149 and hsa-miR-605 gene polymorphisms with gastrointestinal cancer risk. Mol Carcinog 51 (Suppl 1): E21-E31, 2012.

29. Huang SP, Levesque E, Guillemette C, Yu CC, Huang CY, Lin VC, Chung IC, Chen LC, Laverdière I, Lacombe L, et al: Genetic variants in microRNAs and microRNA target sites predict biochemical recurrence after radical prostatectomy in localized prostate cancer. Int J Cancer 135: 2661-2667, 2014.

30. Yin Z, Li H, Cui Z, Ren Y, Li X, Wu W, Guan P, Qian B, Rothman N, Lan Q, et al: Polymorphisms in pre-miRNA genes and cooking oil fume exposure as well as their interaction on the risk of lung cancer in a Chinese nonsmoking female population. Onco Targets Ther 9: 395-401, 2016. 
31. Li J, Tian F, Li D, Chen J, Jiang P, Zheng S, Li X and Wang S: MiR-605 represses PSMD10/Gankyrin and inhibits intrahepatic cholangiocarcinoma cell progression. FEBS Lett 588: 3491-3500, 2014.

32. Chen L, Liu T, Tu Y, Rong D and Cao Y: Cull promotes melanoma cell proliferation by promoting DEPTOR degradation and enhancing cap-dependent translation. Oncol Rep 35 1049-1056, 2016.

33. Hao S, Luo C, Abukiwan A, Wang G, He J, Huang L, Weber CE, Lv N, Xiao X, Eichmüller SB, et al: miR-137 inhibits proliferation of melanoma cells by targeting PAK2. Exp Dermatol 24: 947-952, 2015.

34. Chang X, Zhang H, Lian S and Zhu W: miR-137 suppresses tumor growth of malignant melanoma by targeting aurora kinase A. Biochem Biophys Res Commun 475: 251-256, 2016.

35. Chen QH, Wang QB and Zhang B: Ethnicity modifies the association between functional microRNA polymorphisms and breast cancer risk: a HuGE meta-analysis. Tumour Biol 35 529-543, 2014.
36. Alhasan AH, Scott AW, Wu JJ, Feng G, Meeks JJ, Thaxton CS and Mirkin CA: Circulating microRNA signature for the diagnosis of very high-risk prostate cancer. Proc Natl Acad Sci USA 113: 10655-10660, 2016.

37. Guo ST, Chi MN, Yang RH, Guo XY, Zan LK, Wang CY, Xi YF, Jin L, Croft A, Tseng HY, et al: INPP4B is an oncogenic regulator in human colon cancer. Oncogene 35: 3049-3061, 2016.

38. Bago R, Sommer E, Castel P, Crafter C, Bailey FP, Shpiro N, Baselga J, Cross D, Eyers PA and Alessi DR: The hVps34-SGK3 pathway alleviates sustained PI3K/Akt inhibition by stimulating mTORC1 and tumour growth. EMBO J 35: 1902-1922, 2016.

39. Flaherty KT, Robert C, Hersey P, Nathan P, Garbe C, Milhem M, Demidov LV, Hassel JC, Rutkowski P, Mohr P, et al; METRIC Study Group: Improved survival with MEK inhibition in BRAFmutated melanoma. N Engl J Med 367: 107-114, 2012. 